\title{
QUADRATIC SYSTEMS WITH AN INVARIANT CONIC HAVING DARBOUX INVARIANTS
}

\author{
JAUME LLIBRE AND REGILENE OLIVEIRA
}

\begin{abstract}
The complete characterization of the phase portraits of real planar quadratic vector fields is very far to be completed. As this attempt is not possible in the whole class due to the large number of parameters (twelve, but, after affine transformations and time rescaling, we arrive at families with five parameters, which is still a big number of parameters), many subclasses have been considered and studied. In this paper we complete the characterization of the global phase portraits in the Poincaré disc of all planar quadratic polynomial differential systems having an invariant conic and a Darboux invariant, constructed using only the invariant conic.
\end{abstract}

\section{INTRODUCTION}

Denote by $\mathbb{R}[x, y]$ the ring of the real polynomials in the variables $x$ and $y$, and consider the differential system in $\mathbb{R}^{2}$ given by

$$
\begin{aligned}
& \dot{x}=P(x, y), \\
& \dot{y}=Q(x, y),
\end{aligned}
$$

where $P, Q \in \mathbb{R}[x, y]$. In (1) the dot denotes derivative with respect to the time $t$ and, we define the degree of system (1) as $m=\max \{\operatorname{deg} P, \operatorname{deg} Q\}$.

A quadratic system is a quadratic polynomial differential system as (1) for which $m=2$. The quadratic systems appear in the modeling of many natural phenomena described in different branches of the sciences, and in biological and physical applications. Of course, the quadratic systems became a matter of interest for the mathematicians because after the linear differential systems they are the easiest polynomial differential systems. More than one thousand of papers have been published about quadratic systems. See for instance $[8,9]$ for a bibliographical survey.

Because we want to study quadratic systems, in this paper we always assume that the polynomials $P$ and $Q$ are coprime, otherwise system (1) can be reduced to a linear or constant system by a rescaling of the time variable.

Key words and phrases. quadratic vector fields, Darboux invariant, phase portraits. 2010 Mathematics Subject Classification: Primary 34C05, 34A34. 
The existence of a first integral for a planar differential system is particularly important because it allows to draw the curves containing the trajectories of the differential system. Also it is interesting to know if a differential system has invariants, i.e. first integrals depending on the time. The invariants that here we will study are the Darboux invariants which allow to describe the asymptotic behavior of the solutions of the system, as showed in $[1,6,7]$. A Darboux invariant is a special invariant of the form $f(x, y) e^{s t}$, they are defined in Subsection 2.2.

In [1] the authors charactherize the global phase portraits in the Poincaré disc of all planar Lotka-Volterra quadratic polynomial differential systems having a Darboux invariant. The characterization of all planar polynomial differential systems with a unique invariant algebraic curve given by a real conic and having a Darboux invariant is in [6], but there the authors do not provide the global phase portraits in the Poincaré disc of such differential systems. The authors of this paper in [7] investigated the quadratic systems with invariant straight lines of total multiplicity two (except to the LoktaVolterra systems which were done in [1]) having Darboux invariants and classified their phase portrait in the Poincaré disc.

In this paper we complete the classification of the global phase portraits in the Poincaré disc of quadratic polynomial differential systems with an invariant algebraic conic having a Darboux invariant. From the mentioned results only remains the study of the quadratic systems having an invariant non-degenerate conic and a Darboux invariant.

The normal forms of quadratic systems with an invariant non degenerate conic is given in Proposition 4 of [2] which we reproduce bellow.

Proposition 1. Applying an affine change of coordinates, the real quadratic systems having an invariant non degenerate conic can be written in one of the following forms:

$$
\begin{array}{ll}
\text { (E) } \quad \dot{x}=(a / 2)\left(x^{2}+y^{2}-1\right)+2 y(p x+q y+r), \\
& \dot{y}=(b / 2)\left(x^{2}+y^{2}-1\right)-2 x(p x+q y+r), \\
(\mathrm{CE}) \quad \dot{x}=(a / 2)\left(x^{2}+y^{2}+1\right)+2 y(p x+q y+r), & \\
& \dot{y}=(b / 2)\left(x^{2}+y^{2}+1\right)-2 x(p x+q y+r), \\
(\mathrm{H}) \quad & \dot{x}=(a / 2)\left(x^{2}-y^{2}-1\right)-2 y(p x+q y+r), \\
& \dot{y}=-(b / 2)\left(x^{2}-y^{2}-1\right)-2 x(p x+q y+r), \\
(\mathrm{P}) \quad & \dot{x}=(b / 2) x y-(a / 2)\left(y-x^{2}\right)+p x+q y+r, \\
& \dot{y}=b y^{2}+c\left(y-x^{2}\right)+2 x(p x+q y+r) .
\end{array}
$$

In the statement of Proposition 1 systems $(\mathrm{E}),(\mathrm{CE}),(\mathrm{H})$ and $(\mathrm{P})$ provide the normal forms of the quadratic systems having an invariant real ellipse, complex ellipse, hyperbola and parabola, respectively.

The next theorem provides the characterization of the Darboux invariants of the quadratic systems having an invariant non degenerate conic. We 
remark that we are only considering the Darboux invariants which can be obtained using only the invariant conic, see for more details Proposition 4.

Theorem 2. The following statements hold for the quadratic systems of Proposition 1.

(1) The quadratic systems of type (E), (CE) and (H) of Proposition 1 do not admit a Darboux invariant constructed using only the invariant conic.

(2) Quadratic systems of type (P) of Proposition 1 having a Darboux invariant, constructed using only the invariant parabola, are

$$
\begin{aligned}
& \dot{x}=p x+q y+r, \\
& \dot{y}=c\left(y-x^{2}\right)+2 x(p x+q y+r),
\end{aligned}
$$

where $c, p, q$ and $r$ real parameters. Moreover, its Darboux invariant is

$$
I(x, y, t)=e^{-c t}\left(y-x^{2}\right) .
$$

Theorem 2 shows that the unique quadratic system with an invariant non-degenerate conic having a Darboux invariant, constructed using only the invariant conic, are those having an invariant parabola.

The next result provides the global classification of all phase portraits in the Poincaré disc of the quadratic systems with an invariant parabola having a Darboux invariant.

Theorem 3. The phase portrait in the Poincaré disc of a quadratic polynomial differential system with an invariant parabola having a Darboux invariant, constructed only using the invariant parabola, is topologically equivalent to one of the 10 phase portraits of Figure 1.

The paper is organized as follows. Basic definitions and results necessary to complete the study of the Darboux invariants and of the phase portraits in the Poincaré disc of the quadratic systems are given in section 2 . In sections 3 and 4 we prove Theorems 2 and 3 respectively.

\section{BASIC RESULTS}

2.1. Invariants. If $\Omega$ is an open and dense subset of $\mathbb{R}^{2}$ and $H: \Omega \rightarrow \mathbb{R}$ is a nonconstant function such that $H(x(t), y(t))$ is constant for all the values of $t$ for which $(x(t), y(t))$ is a solution of system (1) contained in $\Omega$, then we say that $H$ is a first integral of system (1) on $\Omega$. So $H$ is a first integral of system (1) if and only if

$$
P \frac{\partial H}{\partial x}+Q \frac{\partial H}{\partial y}=0
$$

for all $(x, y) \in U$.

Let $\Omega$ be an open and dense subset of $\mathbb{R}^{2}$, an invariant of system (1) in $\Omega$ is a nonconstant analytic function $I$ in the variables $x, y$ and $t$ such 


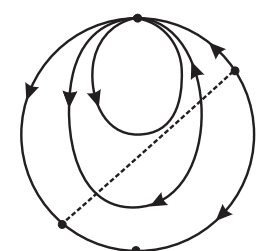

(1)

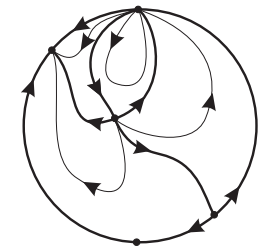

(4)

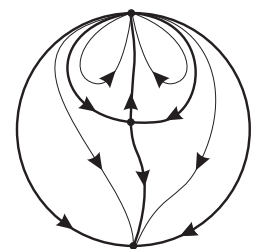

(7)

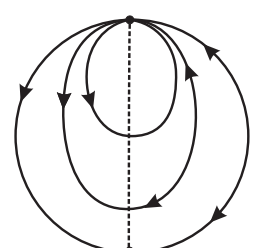

(2)

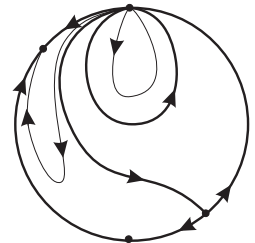

(5)

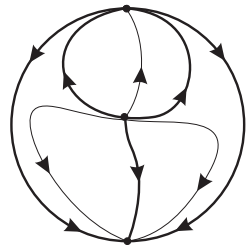

(8)

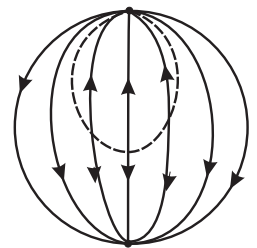

(10)

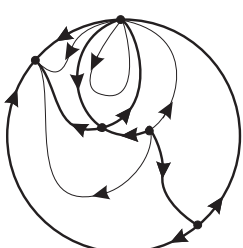

(3)

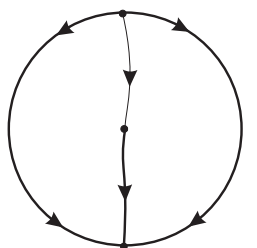

(6)

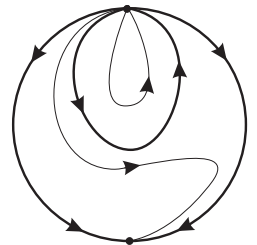

(9)

Figure 1. Global phase portraits of quadratic systems with an invariant parabola and a Darboux invariant. The dashed curves are curves covered of singular points of the system. The thick lines are formed by separatrices of the system, and the thin lines are orbits in the canonical regions of the system.

that $I(x(t), y(t), t)$ is constant on all solution curves $(x(t), y(t))$ of system (1) contained in $\Omega$, i.e.

$$
\frac{\partial I}{\partial x} P+\frac{\partial I}{\partial y} Q+\frac{\partial I}{\partial t}=0
$$

for all $(x, y) \in U$, in other words, $I$ is a first integral of system (1) depending on the time $t$. 
For $f \in \mathbb{C}[x, y]$ the curve $f(x, y)=0$ is an invariant algebraic curve of system (1) if there exists $K \in \mathbb{C}[x, y]$ such that

$$
P \frac{\partial f}{\partial x}+Q \frac{\partial f}{\partial y}=K f
$$

The polynomial $K$ is called the cofactor of the invariant algebraic curve $f=0$. If $K=0$ then $f$ is a polynomial first integral.

Let $f, g \in \mathbb{C}[x, y]$ such that $f$ and $g$ are relatively prime in the ring $\mathbb{C}[x, y]$, or that $g=1$. Then the function $\exp (f / g)$ is a exponential factor of system (1) if there exists a polynomial $L \in \mathbb{C}[x, y]$ of degree at most $m-1$ such that

$$
P \frac{\partial \exp (f / g)}{\partial x}+Q \frac{\partial \exp (f / g)}{\partial y}=L \exp (f / g)
$$

The polynomial $L$ is called the cofactor of the exponential factor $\exp (f / g)$.

2.2. Darboux invariants. A Darboux invariant for system (1) is an invariant $I$ of the form

$$
I(x, y, t)=f_{1}^{\lambda_{1}} \cdots f_{p}^{\lambda_{p}} F_{1}^{\mu_{1}} \cdots F_{q}^{\mu_{q}} e^{s t}
$$

where $f_{i}=0$ are invariant algebraic curves of system (1) for $i=1, \ldots p$, and $F_{j}$ are exponential factors of system (1) for $j=1, \ldots, q, \lambda_{i}, \mu_{j} \in \mathbb{C}$ and $s \in \mathbb{R} \backslash\{0\}$.

The next proposition explain how to find Darboux invariants and it is proved in [3].

Proposition 4. Suppose that a polynomial system (1) of degree $m$ admits $p$ invariant algebraic curves $f_{i}=0$ with cofactors $K_{i}$ for $i=1, \ldots, p, q$ exponential factors $\exp \left(g_{j} / h_{j}\right)$ with cofactors $L_{j}$ for $j=1, \ldots, q$, then, if there exist $\lambda_{i}$ and $\mu_{j} \in \mathbb{C}$ not all zero such that

$$
\sum_{i=1}^{p} \lambda_{i} K_{i}+\sum_{j=1}^{q} \mu_{j} L_{j}=-s,
$$

for some $s \in \mathbb{R} \backslash\{0\}$, then substituting $f_{i}^{\lambda_{i}}$ by $\left|f_{i}\right|^{\lambda_{i}}$ if $\lambda_{i} \in \mathbb{R}$, the real (multivalued) function

$$
f_{1}^{\lambda_{1}} \ldots f_{p}^{\lambda_{p}}\left(\exp \left(\frac{g_{1}}{h_{1}}\right)\right)^{\mu_{1}} \ldots\left(\exp \left(\frac{g_{q}}{h_{q}}\right)\right)^{\mu_{q}} e^{s t}
$$

is a Darboux invariant of system (1).

2.3. Poincaré compactification. The phase portrait of a system is the decomposition of its domain of definition as union of all its orbits.

Let

$$
\mathcal{X}=P(x, y) \frac{\partial}{\partial x}+Q(x, y) \frac{\partial}{\partial y}
$$

be the planar polynomial vector field of degree $m$ associated to the polynomial differential system (1). The Poincaré compactified vector field $\pi(\mathcal{X})$ of 
$\mathcal{X}$ is an analytic vector field induced on $\mathbb{S}^{2}$ as we describe in what follows (for more details see [4]).

Denote by $\mathbb{S}^{2}=\left\{y=\left(y_{1}, y_{2}, y_{3}\right) \in \mathbb{R}^{3} ; y_{1}^{2}+y_{2}^{2}+y_{3}^{2}=1\right\}$ and by $T_{y} \mathbb{S}^{2}$ the tangent plane to $\mathbb{S}^{2}$ at point $y$. We identify $\mathbb{R}^{2}$ with $T_{(0,0,1)} \mathbb{S}^{2}$ and let $f: T_{(0,0,1)} \mathbb{S}^{2} \rightarrow \mathbb{S}^{2}$ be the central projection. The map $f$ defines two copies of $\mathcal{X}$ on $\mathbb{S}^{2}$, one in the southern hemisphere and the other in the northern hemisphere. Denote by $\mathcal{X}^{\prime}$ the vector field $D(f) \circ \mathcal{X}$ defined on $\mathbb{S}^{2} \backslash \mathbb{S}^{1}$, where $\mathbb{S}^{1}=\left\{y \in \mathbb{S}^{2} ; y_{3}=0\right\}$, the equator of $\mathbb{S}^{2}$, is identified with the infinity of $\mathbb{R}^{2}$.

The extension of $\mathcal{X}^{\prime}$ to a vector field $\pi(\mathcal{X})$ on $\mathbb{S}^{2}$, including $\mathbb{S}^{1}$ is the unique analytic extension of $y_{3}^{m-1} \mathcal{X}^{\prime}$ to $\mathbb{S}^{2}$, where $m$ is the degree of $\mathcal{X}$. On $\mathbb{S}^{2} \backslash \mathbb{S}^{1}$ there is two symmetric copies of $\mathcal{X}$. The behavior of $\pi(\mathcal{X})$ near $\mathbb{S}^{1}$ determines the behavior of $\mathcal{X}$ in a neighborhood of the infinity. The Poincaré compactification $\pi(\mathcal{X})$ has $\mathbb{S}^{1}$ as an invariant curve under the flow of $\pi(\mathcal{X})$. Finally, the projection of the closed northern hemisphere of $\mathbb{S}^{2}$ on $y_{3}=0$ under $\left(y_{1}, y_{2}, y_{3}\right) \mapsto\left(y_{1}, y_{2}\right)$ is called the Poincaré disc $\mathbb{D}$, and its boundary is $\mathbb{S}^{1}$.

We say that the vector fields $\mathcal{X}$ and $\mathcal{Y}$ are topologically equivalent if there exists a homeomorphism on $\mathbb{S}^{2}$ preserving the infinity $\mathbb{S}^{1}$ carrying orbits of the flow induced by $\pi(\mathcal{X})$ into orbits of the flow induced by $\pi(\mathcal{Y})$ preserving or not the orientation of the orbits.

For the differentiable manifold $\mathbb{S}^{2}$ we consider six local charts $U_{i}=\{y \in$ $\left.\mathbb{S}^{2} ; y_{i}>0\right\}$ and $V_{i}=\left\{y \in \mathbb{S}^{2} ; y_{i}<0\right\}$, where $i=1,2,3$, and the diffeomorphisms $F_{i}: U_{i} \rightarrow \mathbb{R}^{2}$ and $G_{i}: V_{i} \rightarrow \mathbb{R}^{2}$, for $i=1,2,3$, which are the inverses of the central projections from the tangent planes at the points $(1,0,0),(-1,0,0),(0,1,0),(0,-1,0),(0,0,1)$ and $(0,0,-1)$, respectively. So, we can compute the explicit expression of $\pi(\mathcal{X})$ as follows. Denote by $z=(u, v)$ the value of $F_{i}(y)$ and $G_{i}(y)$, for any $i=1,2,3$, therefore has $z$ different meaning depending on the local charts that we are working. After some computations, we get that $\pi(\mathcal{X})$ is given by

$$
\begin{gathered}
v^{m} \Delta(z)\left(Q\left(\frac{1}{v}, \frac{u}{v}\right)-u P\left(\frac{1}{v}, \frac{u}{v}\right),-v P\left(\frac{1}{v}, \frac{u}{v}\right)\right) \text { in } U_{1}, \\
v^{m} \Delta(z)\left(P\left(\frac{u}{v}, \frac{1}{v}\right)-u Q\left(\frac{u}{v}, \frac{1}{v}\right),-v Q\left(\frac{u}{v}, \frac{1}{v}\right)\right) \text { in } U_{2}, \\
\Delta(z)(P(u, v), Q(u, v)) \text { in } U_{3},
\end{gathered}
$$

where $\Delta(z)=\left(u^{2}+v^{2}+1\right)^{-(m-1) / 2}$. The expressions for $V_{i}$ 's are the same as those for $U_{i}$ 's multiplied by the factor $(-1)^{m-1}$. In these coordinates and for the local charts $U_{1}, V_{1}, U_{2}$ and $V_{2}$ we have that $v=0$ always denotes the points of the infinity $\mathbb{S}^{1}$. 
2.4. $\alpha$ and $\omega$ limit sets and the Darboux invariant. Let $\phi_{p}(t)=$ $(x(t), y(t))$ be the maximal solution of system (1) defined on the interval $\left(\alpha_{p}, \omega_{p}\right)$ such that $\phi_{p}(0)=p$. If $\omega_{p}=\infty$ we define the $\omega$-limit set of $p$ as

$$
\omega(p)=\left\{q \in \mathbb{R}^{2}: \exists\left\{t_{n}\right\} \text { with } t_{n} \rightarrow \infty \text { and } \phi_{p}\left(t_{n}\right) \rightarrow q \text { when } n \rightarrow \infty\right\} .
$$

Analogously, if $\alpha_{p}=-\infty$ we define the set $\alpha$-limit set of $p$ as

$$
\alpha(p)=\left\{q \in \mathbb{R}^{2}: \exists\left\{t_{n}\right\} \text { with } t_{n} \rightarrow-\infty \text { and } \phi_{p}\left(t_{n}\right) \rightarrow q \text { when } n \rightarrow \infty\right\} .
$$

The next proposition, proved in [7], explain how the Darboux invariant provides information about the $\alpha$ - and $\omega$-limit sets of all orbits of system (1).

Proposition 5. Let $I(x, y, t)=f(x, y) e^{s t}$ be a Darboux invariant of system (1). If $p \in \mathbb{R}^{2}$ and $\phi_{p}(t)$ is the maximal solution of system (1) in the interval $\left(\alpha_{p}, \omega_{p}\right)$ such that $\phi_{p}(0)=p$, then

(1) $\omega(p) \subset\{f(x, y)=0\} \cup \mathbb{S}^{1}$ if $\omega_{p}=\infty$,

(2) $\alpha(p) \subset\{f(x, y)=0\} \cup \mathbb{S}^{1}$ if $\alpha_{p}=-\infty$,

where $\mathbb{S}^{1}$ denotes the infinity of the Poincaré disc.

2.5. Separatrices and canonical regions. Let $\mathbb{D}$ be the Poincaré disc and $\pi(\mathcal{X})$ be the Poincaré compactification on $\mathbb{D}$ of the polynomial differential system (1) defined in $\mathbb{R}^{2}$. Consider $\phi$ the analytic flow associated to $\pi(\mathcal{X})$ and denote by $(U, \phi)$ the flow of $\phi$ on an invariant set $U \subset \mathbb{D}$. The flow $(U, \phi)$ is parallel if it is topologically equivalent to one to the following flows:

(i) the flow in $\mathbb{R}^{2}$ given by the differential system $\dot{x}=1, \dot{y}=0$, known as trip flow.

(ii) the flow in $\mathbb{R}^{2} \backslash\{0$,$\} given in polar coordinates by the differential$ system $\dot{r}=0, \dot{\theta}=1$, known as annular flow;

(iii) the flow in $\mathbb{R}^{2} \backslash\{0$,$\} given in polar coordinates by the differential$ system $\dot{r}=r, \dot{\theta}=0$, known as spiral or radial flow.

Moreover, the separatrices of a polynomial vector field $\pi(\mathcal{X})$ in the Poincaré disc $\mathbb{D}$ are all the infinity of $\mathbb{R}^{2}$ (i.e. all orbits of $\pi(\mathcal{X})$ on the boundary of $\mathbb{S}^{1}$ of the Poincaré disc), all the finite singular points of $\pi(\mathcal{X})$, all the limit cycles of $\pi(\mathcal{X})$, and all the separatrices of the hyperbolic sectors of the finite and infinite singular points of $\pi(\mathcal{X})$.

Denote by $\mathbb{S}$ the union of the separatrices of $(\mathbb{D}, \phi)$ defined by $\pi(\mathcal{X})$. $\mathbb{S}$ is an invariant closed set. In addition, if $N$ is a connected component of $\mathbb{D} \backslash \mathbb{S}$, then $N$ is an invariant set under the flow $\phi$, and the flow $(N, \phi \backslash N)$ is known as canonical region of the flow. For a given vector field $\phi(\mathcal{X})$ its separatrix configuration is formed by all the separatrices of $\phi(\mathcal{X})$ plus an orbit in each one of its canonical regions.

The next two results are fundamental in the description of the phase portrait of a flow associated to the planar vector field $\pi(\mathcal{X})$. 
Theorem 6. Suppose that the number of separatrices of the flow $(\mathbb{D}, \phi)$ is finite. Then every canonical region of the flow $(\mathbb{D}, \phi)$ is parallel.

Theorem 7. Let $(\mathbb{D}, \phi)$ and $(\mathbb{D}, \psi)$ be two compactified Poincaré flows with finitely many separatrices generated by the solutions of two polynomial vector fields (3). Then they are topologically equivalent if and only if their separatrix configurations are topologically equivalent.

The proof of the two results above can be found in [5]. From the previous theorem it follows that to classify the phase portrait in the Poincaré disc of a planar polynomial differential system having finitely many separatrices it is enough to describe their separatrix configuration.

\section{DARBOUX INVARIANTS}

Proposition 1 provides the normal forms of all quadratic system with an invariant non degenerate conic. So to prove Theorem 2 we must investigate each system described in Proposition 1.

Proof of Theorem 2 for real or complex ellipses. Let $f_{i}=x^{2}+y^{2}+(-1)^{i}$, $i=1,2$. It is easy to check that $f_{i}$ satisfy the equation

$$
\frac{\partial f_{i}}{\partial x} \dot{x}+\frac{\partial f_{i}}{\partial y} \dot{y}=f_{i} K
$$

for $K=a x+b y$, where $(\dot{x}, \dot{y})$ are given by (E) if $i=1$ and by (CE) if $i=2$. So $f_{1}=0$ is an invariant real ellipse with cofactor $K$ for system (E) and $f_{2}=0$ is an invariant complex ellipse with the same cofactor $K$ for system $(\mathrm{CE})$.

As the equation $\lambda K+s=0$ has no solution for $s \neq 0$, it follows from Proposition 4 that does not exist quadratic systems with invariant real or complex ellipses having a Darboux invariant, constructed only using the invariant ellipse.

Proof of Theorem 2 for hyperbolas. Let $f=x^{2}-y^{2}-1$. Because $f$ satisfy the equation

$$
\frac{\partial f}{\partial x} \dot{x}+\frac{\partial f}{\partial y} \dot{y}=f K
$$

for $K=a x+b y$ where $(\dot{x}, \dot{y})$ are given by $(\mathrm{H}), f=0$ is an invariant hyperbola with cofactor $K$ for systems of type $(H)$ in Theorem 2 .

Observe that $\lambda K+s=0$ has no solution if $s \neq 0$. So from Proposition 4 there are no quadratic systems with invariant hyperbolas having a Darboux invariant, constructed only using the invariant hyperbola.

Proof of Theorem 2 for parabolas. Consider $f(x, y)=y-x^{2}$ and $K(x, y)=$ $a x+b y+c$, we assume $c \neq 0$ otherwise $f$ is a polynomial first integral and 
the phase portrait of the system is easy to do. Then the following equation is satisfied

$$
\frac{\partial f}{\partial x} \dot{x}+\frac{\partial f}{\partial y} \dot{y}=f K
$$

where $(\dot{x}, \dot{y})$ are given by $(\mathrm{P})$. So $f$ is an invariant parabola for system (P) with cofactor $K$. Moreover the solution of equation $\lambda K+s=0$ has an unique solution if $s \neq 0$, the solution is $\lambda=-s / c, a=b=0$. So $I(x, y, t)=e^{s t}\left(y-x^{2}\right)^{-\frac{s}{c}}$ is Darboux invariant for system for every $s, c \in$ $\mathbb{R} \backslash\{0\}$, constructed only using the invariant parabola. Consequently, also $e^{-c t}\left(y-x^{2}\right)$ is a Darboux invariant.

\section{Phase portraits}

In this section we prove Theorem 3 .

Proof of Theorem 3. It follows from the proof of Theorem 2 that a quadratic system having an invariant non-degenerate conic and a Darboux invariant (constructed using only the invariant conic) has an invariant parabola and its normal form is

$$
\dot{x}=p x+q y+r, \quad \dot{y}=c\left(y-x^{2}\right)+2 x(p x+q y+r) .
$$

In order to study the global phase portrait of those systems in the Poincaré disc, first we study the local phase portrait of the finite and infinite singular points according to the following cases.

Case 1: $c=0$. Then system (4) after the change of independent variable $d s=(p x+q y+r) d \tau$ becomes the linear system $x^{\prime}=1, y^{\prime}=2 x$ where the prime denotes the derivative with respect to $s$. The phase portrait of the system $x^{\prime}=1, y^{\prime}=2 x$ is easy to do. Then adding the straight line of singular points $p x+q y+r=0$ we get two non-equivalent phase portraits of system (4) when $c=0$, they are the phase portraits (1) and (2) of Figure 1.

From now on we assume $c \neq 0$. Note that if $q \neq 0$ then we can suppose $q>0$, otherwise we do the change of variables $(x, y, t) \rightarrow(x, y,-t)$.

Case 2: $c \neq 0, q>0$. Denote by $\Delta=p^{2}-4 p r$ and consider the points

$$
\left(\frac{-p \pm \sqrt{\Delta}}{2 q}, \frac{-2 q r+p(p \pm \sqrt{\Delta})}{2 q^{2}}\right) .
$$

If $\Delta>0$ system (4) has two finite singular points and their eigenvalues are $c$ and $-\sqrt{\Delta}$ and, $c$ and $\sqrt{\Delta}$, respectively. Thus, in this case system, (4) has two hyperbolic finite singular points, a saddle and a node, which is stable if $c<0$ and unstable if $c>0$, see for more details Theorem 2.15 of [4].

If $\Delta=0$ system (4) admits a unique finite singular point $(-p /(2 q), r / q)$, that is a saddle-node (see Theorem 2.19 of [4]). Finally, if $\Delta=0$ the system has no finite singular points. 
To study the singular points at infinity of a polynomial vector field via the Poincaré compactification we need to study the singular points on $v=0$ in the local chart $U_{1}$ and the origin of the local chart $U_{2}$, see subsection 2.3.

The Poincaré compactification $\pi(\mathcal{X})$ of $(4)$ in the local chart $U_{1}$ is given by

$$
\begin{aligned}
& \dot{u}=2 p-c+2 q u+2 r v+(c-p) u v-q u^{2} v-r u v^{2}, \\
& \dot{v}=-p v^{2}-q u v^{2}-r v^{3} .
\end{aligned}
$$

Then $(u, v)=((c-2 p) / 2 q, 0)$ is the unique infinite singular point in this chart. As its eigenvalues are 0 and $2 q$, this point is a semi-hyperbolic singular point. Re-writting the system, applying Theorem 2.19 of [4], and using the notation of that theorem we have $\lambda=2, G(y)=-2 c u^{2} / q+\ldots$ and $m=2$. So the point $((c-2 p) / 2 q, 0)$ in the local chart $U_{1}$ is a saddle-node, and the line $u=0$ separates the parabolic sector from the two hyperbolic sectors in the Poincaré disc.

System (4) in the chart $U_{2}$ is written as

$$
\begin{aligned}
& \dot{u}=q v-2 q u^{2}+(p-c) u v+r v^{2}+(c-2 p) u^{3}-2 r u^{2} v, \\
& \dot{v}=-2 q u v-c v^{2}+(c-2 p) u^{2} v-2 r u v^{2},
\end{aligned}
$$

having the origin as a nilpotent equilibrium point because $q>0$. Applying Theorem 3.5 in [4] and using the notation introduced there we have $F(u)=$ $-4 u^{3}+\ldots$ and $G(u)=-6 u+\ldots$ Thus, in this case $m=-3, a=-4, n=1$ and $b=-6$, therefore $b^{2}+4 a(n+1)=4$ and $(0,0)$ is a singular point with a hyperbolic sector and an elliptic sector, as shown in Figure $(k)$ of Theorem 3.5 of [4]. Now we need to decide the position of these sectors in the Poincaré disc, for this we shall do some blow ups.

Doing the blow up $(u, v) \rightarrow(u, w)$, where $u=u$ and $w=v / u$ we get the system

$$
\begin{aligned}
& \dot{u}=-2 q u^{2}+q u w+(c-2 p) u^{3}+(p-c) u^{2} w-2 r u^{3} w+r u^{2} w^{2}, \\
& \dot{w}=-q w^{2}+p u w^{2}+r u w^{3},
\end{aligned}
$$

which has $(0,0)$ as a linearly zero singular point. So applying a second blow up $(u, w) \rightarrow(u, W)$, where $W=w / u$ we get a new system that, after the rescaling of the time by $d s=u d t$ writes as

$$
\begin{aligned}
u^{\prime} & =-2 q u+(c-2 p) u^{2}+q u W+(p-c) u^{2} W-2 r u^{3} W+r u^{3} W^{2}, \\
w^{\prime} & =2 q W+(2 p-c) u W-2 q W^{2}+(c-2 p) u W^{2}+2 r u^{2} W^{2}-2 r u^{2} W^{3},
\end{aligned}
$$

where the prime denotes the derivative with respect to $s$. This system has two singular points with $u=0$. They are $(u, W)=(0,0)$ and $(u, W)=(0,1)$. The eigenvalues of these singular points are $-2 q, 2 q$ and $-2 q,-q$ respectively. Thus the first point is a hyperbolic saddle and the second point is a stable node because $q>0$. From the blow down we get local behavior of the solutions in a neighborhood of $(0,0)$ in the local chart $U_{2}$ when $q \neq 0$. In Figure 2 we describe step by step how we get the local phase portrait of the origin in the local chart $U_{2}$. 


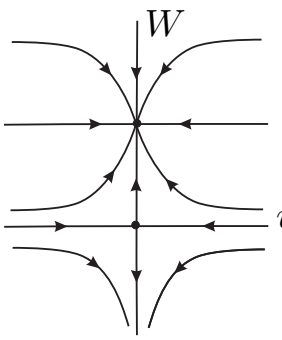

(7)

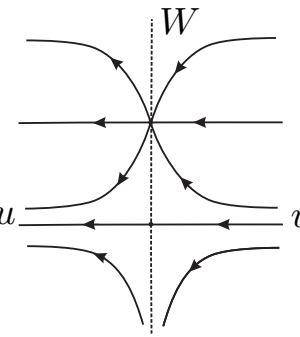

$\left(6^{\prime}\right)$

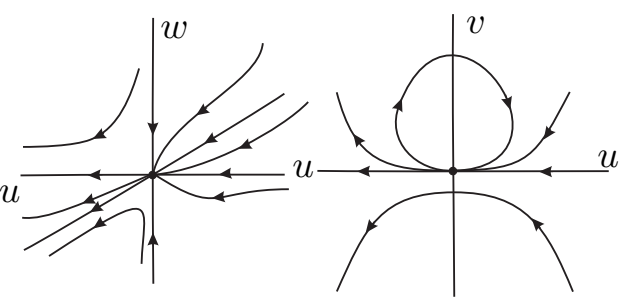

(6)

(5)

Figure 2. Blow down of the origin in the local chart $U_{2}$.

Finally we observe that each finite singular point, when they exist, is located on the invariant parabola $y-x^{2}=0$, so the system has no limit cycles. Indeed, if a limit cycle exists there will be a singular point in the bounded region limited by it, see for instance Theorem 1.31 of [4], but since here all the finite singular points are in the parabola, limit cycles cannot surround such equilibria. So in this case playing with the different values of the parameters $p, q, c$ and $r$ there are three distinct phase portraits which are topologically equivalent to the phase portraits (3), (4) and (5) of Figure 1.

Case 3: $q=0$ and $\operatorname{cpr}(c-2 p) \neq 0$. Then system (4) is

$$
\dot{x}=p x+r, \quad \dot{y}=c\left(y-x^{2}\right)+2 x(p x+r) .
$$

Then $\left(-r / p, r^{2} / p^{2}\right)$ is the unique finite singular point of system (8) and its eigenvalues are $c$ and $p$. So if $c p<0$ the finite singular point is a hyperbolic saddle, and if $c p>0$ it is a hyperbolic node (stable if $c+p<0$ and unstable if $c+p>0$ ), for more details see Theorem 2.15 of [4].

In the local chart $U_{1}$ the compactified vector field $\pi(\mathcal{X})$ of $(8)$ writes

$$
\begin{aligned}
& \dot{u}=2 p-c+2 r v+(c-p) u v-r u v^{2}, \\
& \dot{v}=-p v^{2}-r v^{3},
\end{aligned}
$$

so this system has no infinite singular points on this local chart.

The compactified vector field $\pi(\mathcal{X})$ of $(8)$ in the local chart $U_{2}$ is given by

$$
\begin{aligned}
& \dot{u}=(p-c) u v+r v^{2}+(c-2 p) u^{3}-2 r u^{2} v, \\
& \dot{v}=-c v^{2}+(c-2 p) u^{2} v-2 r u v^{2},
\end{aligned}
$$

so the origin is a linearly zero singular point. In order to identify the local phase portrait of this degenerate point we apply the blow up $(u, v) \rightarrow(u, w)$, where $w=v / u$ and get the following system after the rescaling of the time $d s=u d t$

$$
\begin{aligned}
& u^{\prime}=(c-2 p) u^{2}+(p-c) u w-2 r u^{2} w+r u w^{2}, \\
& w^{\prime}=-p w^{2}+r w^{3},
\end{aligned}
$$

that has two singular points when $u=0$, the points $(0,0)$, a linearly zero singular point and the point $(0,-p / r)$ with eigenvalues $c p / r$ and $-p^{2} / r$. In 
this case, the point $(0,-p / r)$ is a hyperbolic saddle point if $c p>0$, and a hyperbolic node (unstable if $r<0$ and stable if $r>0$ ), otherwise.

Now we apply a second blow up to study the origin. Taking $(u, w) \rightarrow$ $(u, W)$, where $W=w / u$, we get the following system that after rescaling of the time $d \tau=u d s$ becomes

$$
\begin{aligned}
& u^{\prime}=(c-2 p) u+(c-p) u W+2 r u^{2} W-r u^{2} W^{2}, \\
& W^{\prime}=(2 p-c) W-(2 p-c) W^{2}+2 r u W^{2}-2 r u W^{3},
\end{aligned}
$$

where the prime denotes the derivative with respect to $\tau$. This system has two singular points on the line $u=0$, they are $(0,0)$ and $(0,1)$. The eigenvalues of these points are $c-2 p,-(c-2 p)$ and $c-2 p,-p$, respectively. So, the first point is a hyperbolic saddle and, the second is a hyperbolic saddle, if $(c-2 p) p>0$ and a hyperbolic node, otherwise. By the blow down we conclude that the local phase portrait of the origin of the local chart $U_{2}$ is given in Figures 3 and 4 according to the given conditions.

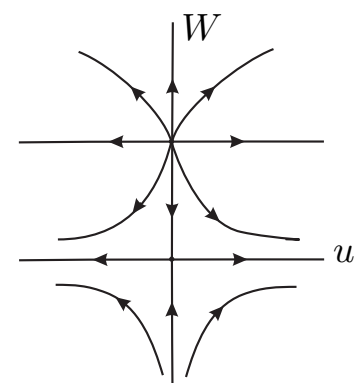

(12)

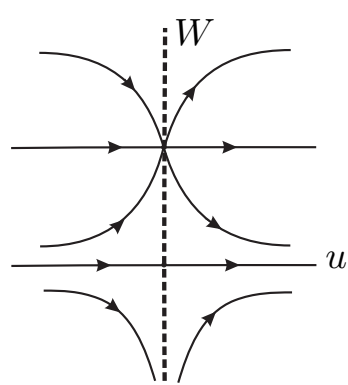

$\left(12^{\prime}\right)$

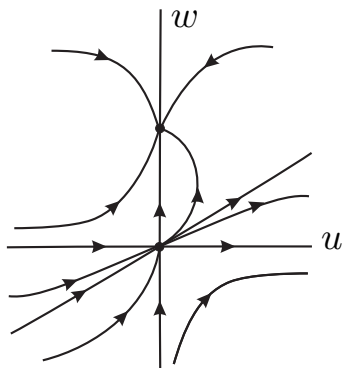

(11)

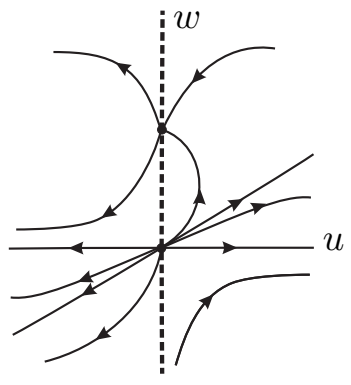

$\left(11^{\prime}\right)$

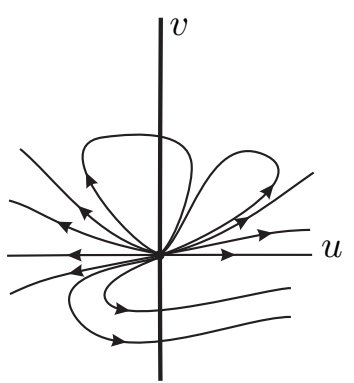

(10)

FiguRE 3. Blow down of the origin in the local chart $U_{2}$, for $p<0, r>0, c>0$ and $c-2 p<0$.

From the previous considerations and observing that the unique finite singular point in this case belongs to the parabola $y-x^{2}=0$ (so the system does not admit limit cycles) we get that the global phase portrait in the Poincaré disc of system (8) under the conditions $q=0$ and $\operatorname{cpr}(c-2 p) \neq 0$ are the phase portraits (6), (7) and (8) of Figure 1. 


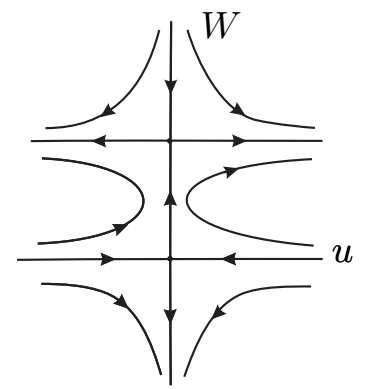

(12)

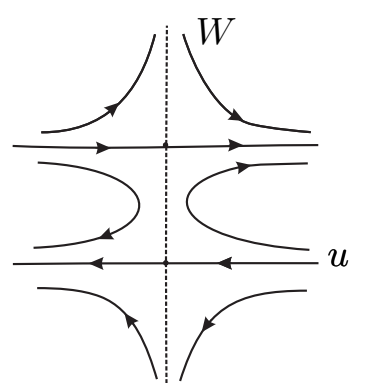

$\left(12^{\prime}\right)$

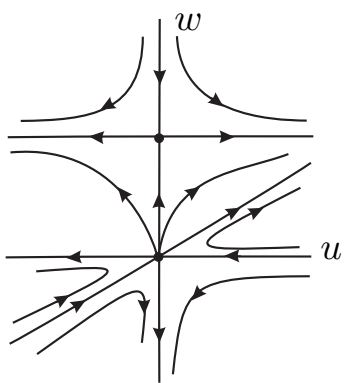

(11)

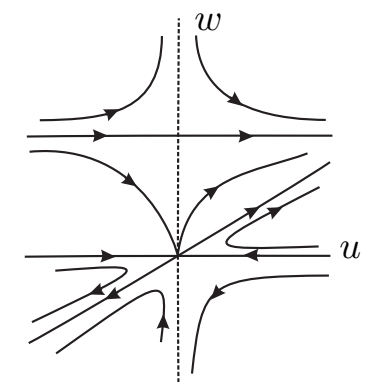

$\left(11^{\prime}\right)$

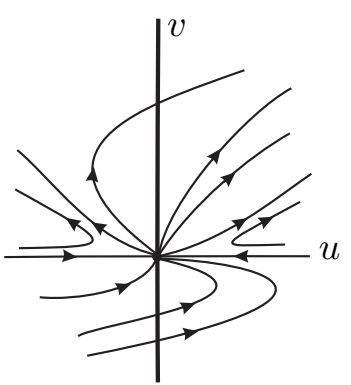

(10)

Figure 4. Blow down of the origin in the local chart $U_{2}$, for $p<0, r>0, c<0$ and $c-2 p>0$.

Case 4: $q=r=0$ and $c(c-2 p) \neq 0$. The study of this case is completely similar to Case 3 , obtaining the same phase portraits.

Case 5: $q=0, c p \neq 0$ and $c=2 p$. In this case system (4) is given by $\dot{x}=r+p x, \dot{y}=2(r+p y)$. Since this system is not quadratic we do not consider this case.

Case 6: $p=q=0, c r \neq 0$. In this case system (4) is written as $\dot{x}=r$, $\dot{y}=2 r x+c y-c x^{2}$. This is a regular system in the plane, without infinite singular points in the local chart $U_{1}$ and with a linearly zero point at the origin of the local chart $U_{2}$. The local phase portrait of this linearly zero point can be studied as the origin of the local chart $U_{2}$ in the Cases 3 and 4 , after two blow ups we get two singular points on the line $u=0,(0,0)$ and $(0,1)$. Their eigenvalues are $-c, c$ and $c, 0$. So we have a hyperbolic saddle and a semi-hyperbolic point. It is not difficult to verify that this point is a saddle-node, because $r \neq 0$. By blow down we get the local phase portrait at origin in this case, as shown in Figure 2. Finally, this system cannot have limit cycles because there are no finite singular points. Therefore we get that the global phase portrait in the Poincaré disc for system (4) under the hypothesis $p=q=0, c r \neq 0$, is topologically equivalent to the phase portrait (9) of Figure 1. 
Case 7: $q=p=r=0$ and $c \neq 0$. Under these conditions, system (4) is given by $\dot{x}=0, \dot{y}=c\left(y-x^{2}\right)$, which is topologically equivalent to system $\dot{x}=0, \dot{y}=1$, except by the points in the parabola $y-x^{2}=0$. In this case the parabola is a curve covered by singular points. So the global phase portrait is topologically equivalent to the phase portrait (10) of Figure 1.

Finally, since system (4) has the Darboux invariant $I(x, y, t)=e^{-c t}\left(y-x^{2}\right)$ all their orbits have $\omega$ - and $\alpha$-limit sets contained in the boundary of the Poincaré disc or to the equilibrium points contained in the parabola $y-x^{2}=$ 0 .

\section{ACKNOWLEDGEMENTS}

The first author is partially supported by a MINECO grant MTM201340998-P, an AGAUR grant number 2014SGR-568, the grants FP7-PEOPLE2012-IRSES 318999 and 316338, the MINECO/FEDER grant UNAB13-4E1604, and a CAPES grant number 88881.030454/2013-01 from the program CSF-PVE. The second author is partially supported by the joint projects FP7-PEOPLE-2012-IRSES numbers 316338, CAPES grant number 88881.030454/2013-01 from the program CSF-PVE, CNPq grant "Projeto Universal 472796/2013-5" and FAPESP grant "Projeto Temático" 2014/00304-2.

\section{REFERENCES}

[1] Y. Bolaños, J. Llibre And C.VAlls, Phase portraits of quadratic Lotka-Volterra Systems with a Darboux invariant in the Poincaré disc. Communications in Contemporary Mathematics 16 (2014), 1350041 (23 pages).

[2] L. Cairó AND J. LliBRe, Darbouxian first integrals and invariants for real quadratic systems having an invariant conic, J. Phys. A: Math. Gen. 35 (2002), 589 - 608.

[3] C. J. Christopher AND J. LliBRe, Integrability via invariant algebraic curves for planar polynomial differential systems, Ann. Differ. Equ. 16 (2000), 5-19.

[4] F. Dumortier, J. Llibre And J. C. Artés, Qualitative theory of planar differential systems, Universitext, Springer, New York, 2006.

[5] D. A. Neumann, Classification of continuous flows on 2-manifolds, Proc. Amer. Math. Soc. 48 (1975), 73-81.

[6] J. Llibre, M. Messias And A. C. Reinol, Darboux invariants for planar polynomial differential systems having an invariant conic, Z. Angew. Math. Phys. 65 (2014), 1127-1136.

[7] J. LliBRe AND R. Oliveira, Quadratic systems with invariant straight lines of total multiplicity two having Darboux invariants, Communications in Contemporary Mathematics 17 (2015), 1450018 (17 pages). 
[8] J. W. REYN, A bibliography of the qualitative theory of quadratic systems of differential equations in the plane, Delf University of Technology, http://ta.twi.tudelf.nl/DV/Staff/ J.W.Reyn.html, 1997.

[9] Ye YanqIan, Theory of Limit Cycles, Translations of Math. Monographs, Vol. 66, Amer. Math. Soc, Providence, 1986.

Departament de Matemàtiques, Universitat Autònoma de Barcelona, 08193 Bellaterra, Barcelona, Catalonia, Spain

E-mail address: jllibre@mat.uab.cat

Departamento de Matemática, Instituto de Ciências Matemáticas e Computação, Universidade de SÃo Paulo, C.P 668, 13566-590, SÃo Carlos, SP, Brazil.

E-mail address: regilene@icmc.usp.br 